\title{
Information Technology for Communities: Development of a Web-based 3D Visualization and Cluster Computing System for Disaster Management
}

\author{
Dr. Ge Jin, Purdue University Calumet (College of Technology)
}

Ge Jin, D.Sc, is currently an assistant professor in the Department of Computer Information Technology and Graphics at the Purdue University Calumet. He teaches computer game development, computer graphics and animation, as well as computer information technology courses at the undergraduate and graduate levels. Prior to joining Purdue University Calumet, he was a postdoctoral research scientist at the George Washington University, Department of Computer Science. Professor Jin holds a B.S. in Computer Science from Peking University, China, and an M.S. in Computer Science from Seoul National University, South Korea. He earned his Doctor of Science degree in Computer Science with a concentration in computer graphics from the George Washington University. His research spans the fields of computer graphics, virtual reality, computer animation, medical visualization, and educational game development. $\mathrm{He}$ is a member of the ACM SIGGRAPH, ASEE, and International Society of Virtual Rehabilitation.

\section{Mr. Gerald A Dekker Jr., Purdue University Calumet}

Gerald Dekker obtained his Master's degree in Computer Science from Purdue University Calumet in 2012. He currently works at the Purdue Calumet Center for Innovation through Visualization and Simulation as a post-graduate researcher in virtual reality and computer graphics.

\section{Mr. John Moreland, Purdue University Calumet}

John Moreland is senior research scientist at the Center for Innovation through Visualization and Simulation at Purdue University Calumet. He has MS in Technology, BS in Computer Graphics Technology, and is currently working on a $\mathrm{PhD}$ in Technology focusing on the application of mixed reality technologies to education.

\section{Prof. Barbara Jean Nicolai, Purdue University Calumet}




\title{
Information Technology for Communities: Development of a Web-based 3D Visualization and Cluster Computing System for Disaster Management
}

\author{
Authors \\ Institution \\ Address
}

\begin{abstract}
Natural disasters can cause huge loss of life, enormous amounts of property damage to the local communities. Although it is impossible to avoid the natural disasters, human sufferings can be reduced by adopting information technologies to the disaster response missions. In this paper, a web-based 3D visualization and cluster computing system was developed to facilitate and expedite the resource distribution process during a disaster. Our disaster management system utilizes state-of-the-art computing cluster with 16 nodes of Intel Xeon-E5 processors (16 cores per node) to process the emergency supply requests from the disaster victims, and calculate the optimal resource distribution routes with the consideration of the damaged transportation infrastructures. The optimized resource distribution problem was solved with distributed all pair shortest path algorithm and the vehicle routing algorithm. The web-based 3D visualization system was developed with Google earth engine to display the disaster areas, affected households and resource distribution routes. The computation result from the cluster was automatically uploaded to the web-based 3D visualization system, enabling the users to immediately see the optimal resource distribution routes in a virtual 3D environment. The visualization system is flexible and can be easily adapted to a Google earth enabled mobile devices, desktop monitors as well as a Cave automatic virtual environment (CAVE). A historical disaster data from the Northwest Indiana was used to demonstrate the functionalities of the developed system.
\end{abstract}

\section{Introduction}

The sights and sounds of disasters and expectation of rapid response is putting increasing pressures on governments and other institutions to move ever more quickly, whether it be in disaster relief endeavors such as the 2004 Asian tsunami, the Katrina hurricane along the Gulf Coast of the United States in 2005, the earthquake in Haiti in 2010, or the most recently earthquake and tsunami in Japan. From the White House report on Hurricane Katrina, the state and local government should develop modern, flexible and transparent logistic system for stockpiling commodities and supplies during the emergency [1]. Improvements in emergency management information and decisions will have a positive impact on those most affected by the disaster - the affected population. The affected population, however, can create difficulties for the decision-maker. Each has varying disaster-caused or exacerbated needs and varied demands for services from the response organizations. With a general perception of lack of planning and coordination on both federal and state levels, the question arose as to the preparedness of local government programs. Indiana Townships are part of state and local government systems which supply indigent populations with needed services. Research has shown that, at present, many systems lack an emergency plan capable of meeting immediate needs such as basic shelter, food, 
clothing, and medical services for a large number of people in a short amount of time [2-6]. Better information and decision support to the manager can provide more accurate focus and priority to balance the emergency response. Geospatial Information System (GIS) based risk assessment and loss estimation tool has been widely used in federal and local government level. Federal Emergency Management Agency (FEMA) has distributed HAZUS loss estimation tool on top of the integrated geographic information system platform ArcGIS (ESRI). It has been used to estimate the damages made by earthquake and flooding [7, 8]. Based on Lesson-learned research from Hurricane Katrina, federal, state and local agencies need to maintain locallyaccurate GIS data that will enhance effective implementation of geospatial technologies for disaster management.

High performance computing has been utilized for a variety of damage estimation and simulation in disaster management applications. Akhter et al. extended open source GRASS (Geographic Resources Analysis Support System) library with MPI and OpenMP framework to perform distributed processing of satellite images for rapid damage severity and extent estimation [9, 10]. Roy et al. indicated that in the emergency management of the real-time data, it is important to adopt distributed geo-data processing methodology for effective decision making [11]. Luis et al. reviewed numerous articles in disaster relief routing and suggested to utilize high performance computing approach for disaster relief routing [12]. Recently, Lecron et al. and Cruz-Chávez et al. exploited grid computing method in solving Vehicle Routing Problem [13, 14].

This paper introduces a GIS based disaster data management, cluster computing and 3D visualization system that focuses on North West Indiana region. The web-based disaster data management and communication system can provide communication between local authorities and indigent population affected by the disaster. The High Performance Computing Cluster will provide expedited disaster management plans for decision makers. The Cluster Computing system gathers the data during a disaster and disburses that information to the administrators who must make critical decisions to provide emergency food, water, medical and rescue distribution. 3D Geospatial Information Visualization system provides situational awareness and a common operating picture of local disaster areas. The local government agencies can visually acquire disaster related information and make appropriate decisions based on the proposed disaster management and decision support system. The visualization system is flexible and can be easily adapted to a Google earth enabled mobile devices, desktop monitors as well as a Cave Automatic Virtual Environment (CAVE). Although the proposed research focuses on the Northwest Indiana region, the generality of the proposed methods and developed prototype system can be easily applied to other regional government agencies across the nation

\section{Web-based Disaster Management and Communication System}

The disaster management and communication module consists of a software/hardware solution. The software consists of a SQL Server database and user interface built on the ASP.NET framework. It contains modules for collecting client information and resource information for services such as housing, transportation, emergency assistance, clothing, etc. The resource inventory data can be used by the Homeland Security Incident Commander in charge of providing support to the affected disaster area. The networking and hardware portion of this system consists of wireless handheld devices or laptops, which can be brought to a disaster site 
to collect indigent demographic information. This information is then added to the SQL database through a wireless network system. Built on a Windows Server platform, this system was implemented on both portable computers and workstations, which can be easily transported if necessary. The Enhanced Entity Relationship model describes the relationships between the agency, resources, clients, and service providers during emergencies. The DMCS system was setup as a two tier application with application screens and class objects residing on a web server and the data being stored in a separate SQL server database. The Web server uses HTTPS security protocol to encrypt the data communication between the client computer and web server. The application requires a login id and password validated through the database to get access to the application. The communication between the DMCS and the database was setup with one connection setup in the web.cfg file. The security of the data is also enhanced by the use of SQL stored procedures that reside on the database for getting and sending data to the web application from the database.

Another feature of DMCS system is a GIS module, which could be used to display geographic conditions. To obtain the latitude and longitude values of an entered address the system uses a web service provided by "Yahoo PlaceFinder". This service can convert an address such as "1600 Pennsylvania Avenue, Washington, DC" into geographic coordinates (Specifically latitude 38.898717, longitude -77.035974).

Figure 1 displays web-based DMCS system described above. Once the disaster manager is assigned to the operation, they begin to collect information about the event in order to inform their actions. The geospatial information is part of the situational awareness needed to develop the common operating picture.

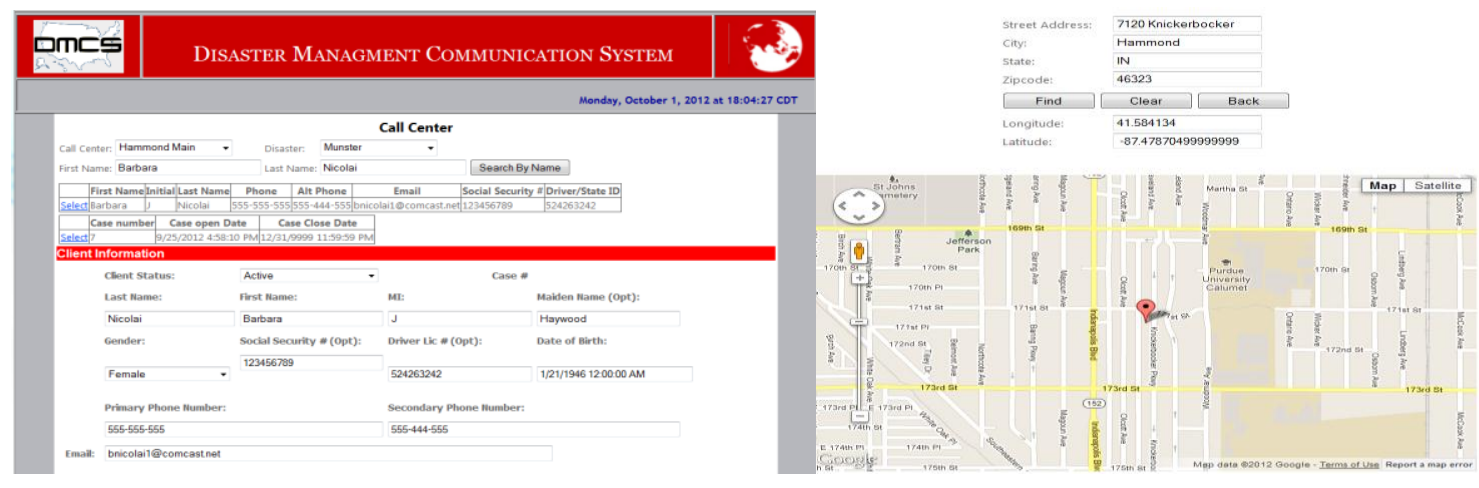

Figure 1. Web-based DMCS Interface and Geo-location API

\section{Automated data transmission between the web-based DMCS and Computing Cluster}

The automated data transmission subsystem was developed to create a secure channel that connects the database to the remote computing cluster. The subsystem is located at the DMCS database server and runs synchronous operations. The data transmission system scans the RDBMS tables in the disaster database to search new requests from the customers. When the new requests matched, the subsystem extracts and collects the data through SQL scripts and writes them into a pre-defined file format. The data includes the information about the resource center and the household (customer demands) affected by the disaster. Afterwards, the subsystem 
automatically connects to the Linux-based supercomputing cluster using SSH protocol. After connecting to remote cluster, it sends the shell command to calculate the optimized resource distribution routes. The data transmission subsystem will continue to check the completion of submitted computation jobs in the computing cluster. Once the job is completed, the subsystem will send the computation result back to the web based DMCS database server. The key functionalities of automated data transmission subsystem include: Automated data transmission using SSH, Extraction of data from the DMCS database with SQL scripts, Automatic conversion of a street address to geographic coordinates and remote invocation and monitoring of jobs inside cluster with SSH shell programming.

\section{Optimized resource distribution route computation using Computing Cluster}

It is essential to provide efficient resource distribution strategy to the decision makers after a disaster impact. A resource distribution center has certain capacities of resources including food, water, clothes, emergency medicines and transportation units. An affected household has certain requests with food, water, clothes and emergency medicines. With the assumption that the amount of resource is sufficient to serve all the affected population, minimizing the resource distribution time is equivalent to minimize the sum of the distance between the resource center and household locations. Therefore, the problem becomes minimizing sum of traveling distance between resource centers to household locations. Minimizing sum of traveling distance with the constraint of the capacity is a vehicle routing problem and can be solved by all pair shortest path algorithm and vehicle routing algorithm. Both all pair shortest path and vehicle routing problem are fundamentally graph problem. In this paper, the graph being processed is the road network structure in North West Indiana region. The GIS road network data was acquired from the US Census Bureau. Unfortunately, the GIS road data is only a collection of line segments that does not provide any connectivity information. To solve this problem, a scan conversion algorithm was implemented to rasterize the GIS road data and recreated adjacent graph structure. The scan conversion algorithm rasterizes unique road color codes for road segments and identifies intersection points (Figure 2). This rasterizer is used to generate an adjacency road network graph to calculate all pair shortest path and vehicle routing problem.

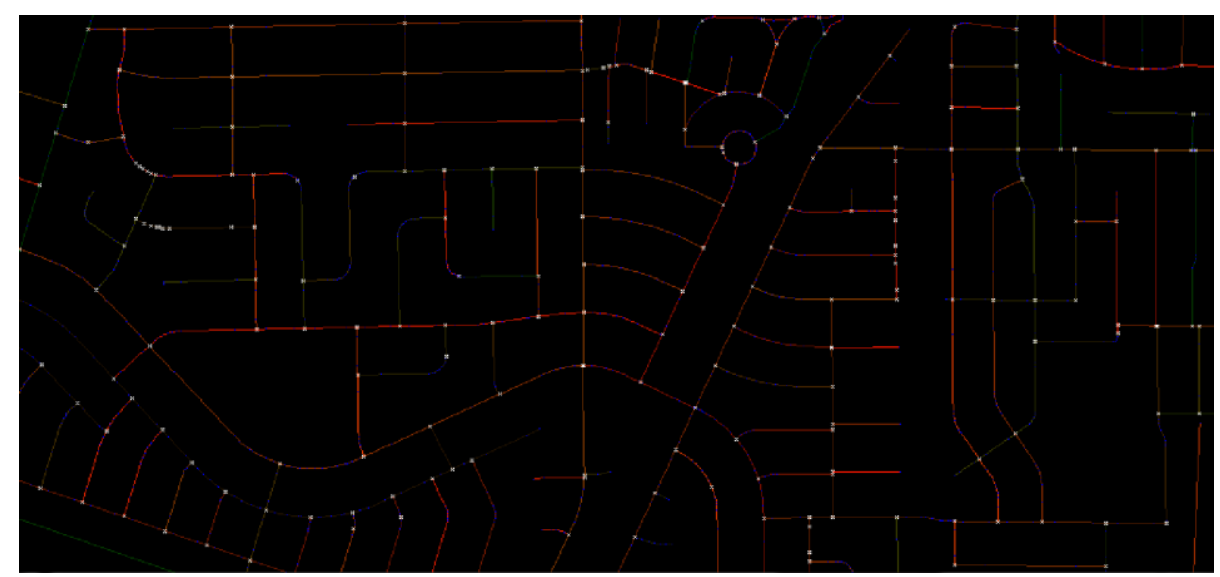

Figure 2. Scan Conversion Algorithm to Generate Road Network Graph 
All pair shortest path algorithm is a graph analysis algorithm to find shortest paths between any two nodes in a weighted graph. Original Floyd-Warshall algorithm is a dynamic programming algorithm that finds the summed weights of the shortest paths between all pairs of vertices, but it does not provide the transition route [15]. In this paper, a distributed Floyd-Warshall algorithm was implemented to provide transition route information inside 16-node computing cluster. The detailed distributed all-pair shortest algorithm is listed in Figure 3 below.

//Distributed Floyd-Warshall all-pair shortest path algorithm

//int num_rows: number of rows in adjacency matrix, float *block: partial //adjacency matrix allocated to a cluster node, int *P: precedence matrix, int //my_rank: cluster node rank, int SIZE: size of adjacency matrix

float* Floyd_1d(int num_rows, float *block, int *P, int my_rank, int SIZE) \{

int $\mathrm{n}=\mathrm{SIZE}$

//Allocate temporary array "row" with the number of vertices in graph

float $*$ row $=($ float $*)$ malloc $(\mathrm{n} * \operatorname{sizeof}($ float $))$;

// Loop over each row in the adjacency matrix

for (int $\mathrm{k}=0 ; \mathrm{k}<\mathrm{n} ; \mathrm{k}++$ )

\{

If the kth row was allocated to this cluster node

Assign kth row from "block" matrix to temporary "row" array

//Broadcast "row" array to all other nodes

MPI_Bcast(row, n, MPI_FLOAT, p, MPI_COMM_WORLD);

//Perform algorithm on block matrix

for (int $\mathrm{i}=0 ; \mathrm{i}<$ num_rows; $\mathrm{i}++$ )

for (int $\mathrm{j}=0 ; \mathrm{j}<\mathrm{n} ; \mathrm{j}++$ )

\{

Compare current distance between ith and jth node.

If the distance passing kth vertex is shorter than current distance, update block matrix and precedence matrix. \}

free(row);

return block;

\}

Figure 3. Distributed Floyd-Warshall algorithm 
The distributed all-pair shortest path algorithm works as follows: each cluster node will obtain a part of the adjacency graph matrix. The shortest path computation will perform in row-first order. Each node will choose the row that will be shared by multiple nodes, and broadcast it to other nodes. These are candidate transition route that if the cumulated distance of passing this graph vertex is shorter than current cumulated distance, then replace the shortest path, and put the transition graph vertex into the precedence matrix $P$.

The vehicle routing problem (VPR) is to construct a minimum cost set of routes that meets all customer demand while minimizing the total distance of transition routes. Because of the difficulty in solving the VRP and additional constraints, VRP problem of realistic size was typically solved via heuristic methods. In this project, an open source $\mathrm{C}++$ package(VRPH) was adopted to solve the vehicle routing problem. VRPH was developed Chris Groer, and provides a modular, well-documented library of local search heuristics for generating solutions to the VRP [16]. The all-pair shortest path matrix will be used as an input to the VRPH software, and it will generate optimized resource distribution route. The optimal distribution route starts from the command center, follow the shortest path to the affected household and resource distribution locations and return back to command center. Different colors indicate the multiple resource distribution routes based on the numbers and capacities of the mobile unit. Figure 4 shows a visualization of the computed resource distribution route in web-based $3 \mathrm{D}$ visualization system.

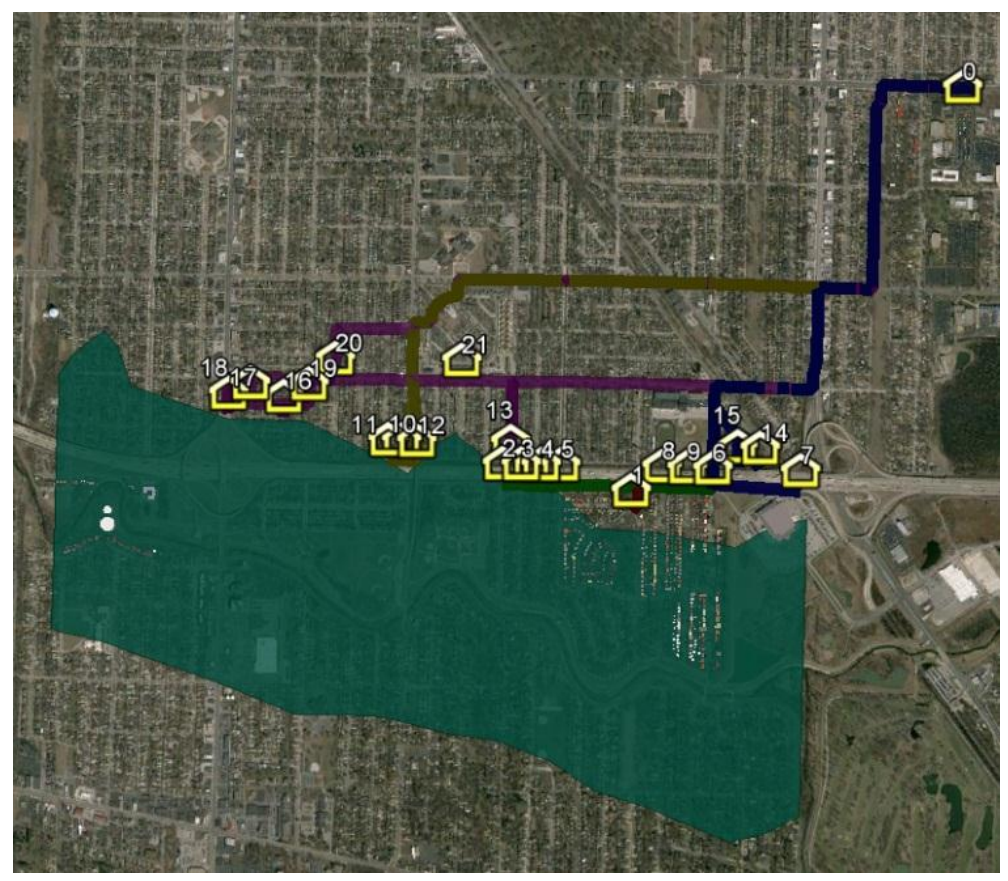

Figure 4. Optimal Route Calculation using VRPH method

The optimal resource distribution route computation utilizes state-of-the-art computing cluster with 16 nodes of Intel Xeon-E5 processors to process the emergency supply requests from the disaster victims, and calculate the optimal resource distribution routes with the consideration of the damaged transportation infrastructures. For the Munster and Hammond city, the HPC can 
update the optimal route in less than 1 minute. Considering the flooded area in the Lake County Indiana, the route computation took 15 minutes inside High Performance Computing Cluster.

\section{D Visualization of the Optimal Resource Distribution Route and Disaster Area}

The computation result from the computing cluster is automatically uploaded to a Google-Earth based 3D visualization system. This approach enables the users to immediately see the computation result from a web based visualization system. The Google Earth software package was chosen as it was both a freely available and reasonably capable GIS suite. Initial testing indicated that selective culling of models would be necessary within the displayed area to insure program responsiveness. Google Earth supports a large subset of the KML (Keyhole Markup Language) specification. A significant feature of the KML specification used in this visualization system is the Network Link element. A KML Network Link allows a Google Earth context to be updated periodically from a remote server. The visualization interface initially loads all possible categories of displayable elements as Network Links. The response files are dynamically generated using PHP and a database backend to reflect the current state of the emergency scenario. Objects which are dynamically generated from database for display includes: Emergency relief packages sources (EMS centers) as KML Models, Emergency relief package destinations as KML Placemarks, Extent of flooded water bodies as KML Polygons, Calculated optimal package delivery routes as KML Lines and Observer photographs inserted as geolocated KML PhotoOverlays. The web based 3D visualization system provides monoscopic and stereoscopic page, which facilitates the usage of both regular monitor and the stereoscopic displays such as 3D monitor and CAVE virtual environment (Figure 5).

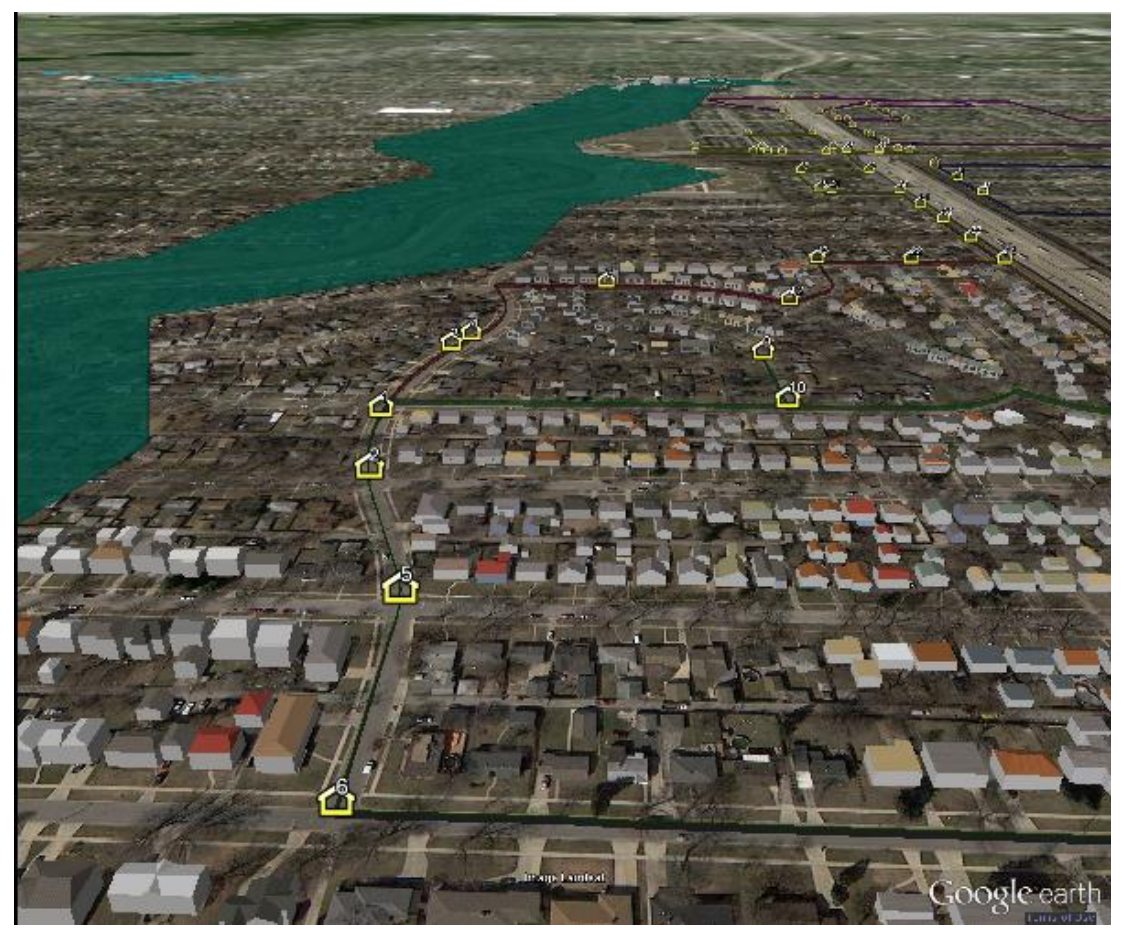

Figure 5. 3D Visualization of 2008 Flood Data in Munster Indiana 
During the disaster, users can upload the photos of disaster areas using mobile and handheld devices. Users can take photo in the field with mobile device. The picture info and data could be acquired from phone instrumentation. The photo and geospatial information was uploaded to server, and pictures will be automatically updated and placed within the Google Earth interface (Figure 6). Google Earth user interface which is dynamically updated or populated with delivery targets, routes, and road obstruction visual indicators via a remote database and compute cluster backed web server.
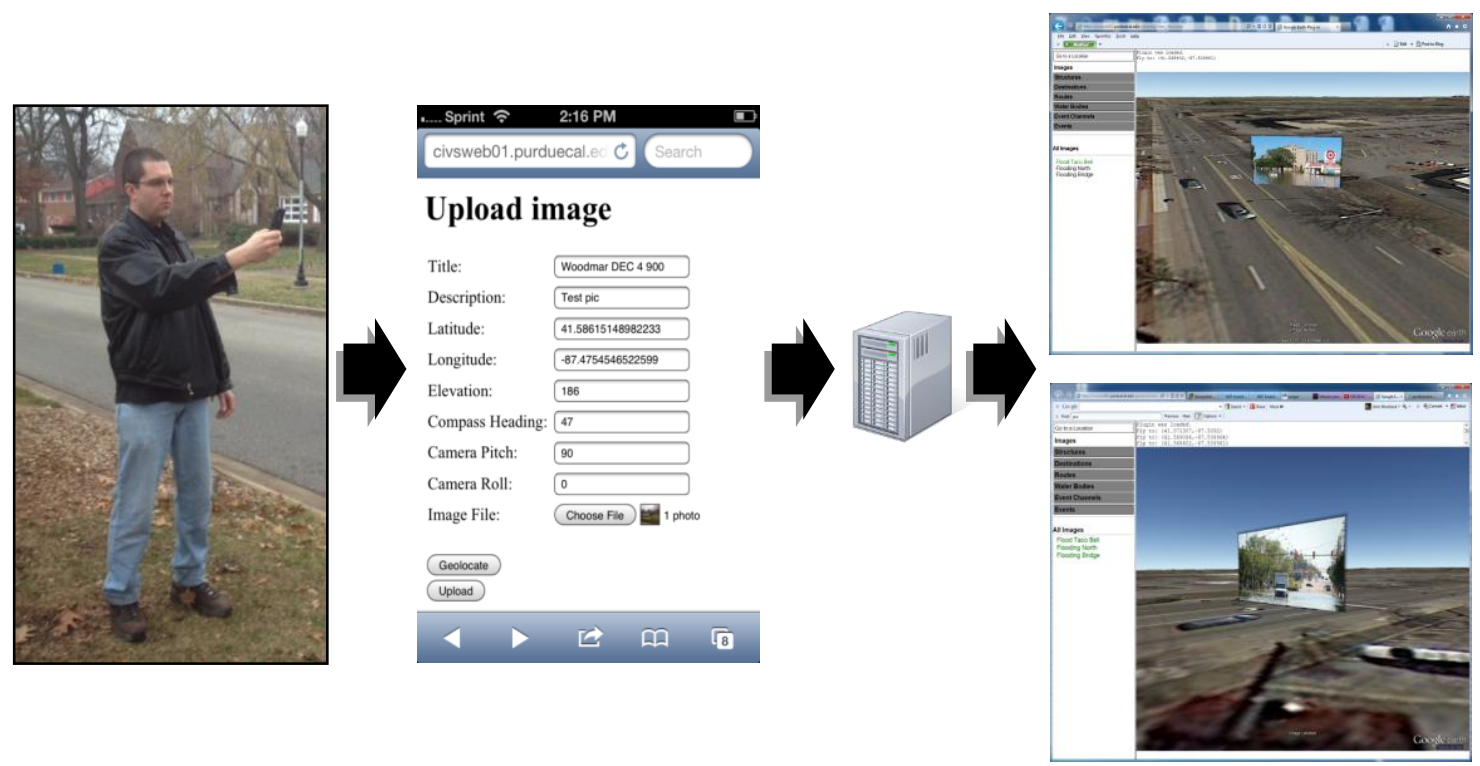

Figure 6. Real-time Update of Disaster Related Photos from Mobile Devices

To test the developed DMCS system, a historical flood data was acquired from FEMA report on 2008 Northwest Indiana flood case. The blue area in the Figure 7 left image is the flooded area along the little calumet river in the Munster city. The flooded area was imported to the HPC and web-based visualization system to compute the optimal resource distribution route.

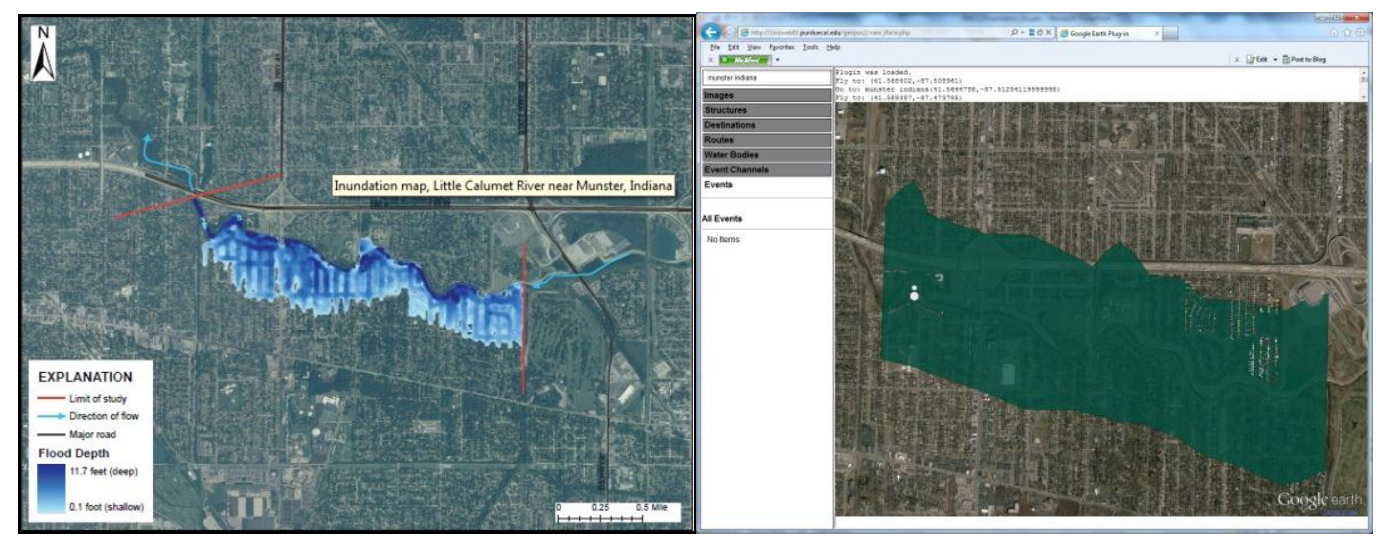

Figure 7. 2008 Flood Data in Munster Indiana 
The flooded area is rapidly changing during a disaster. The high performance computing cluster is capable of dynamically update the optimal resource distribution route with the changes in the flooded area. Figure 8 shows the expansion of the flooded area and resource distribution routes to the affected households over the time.

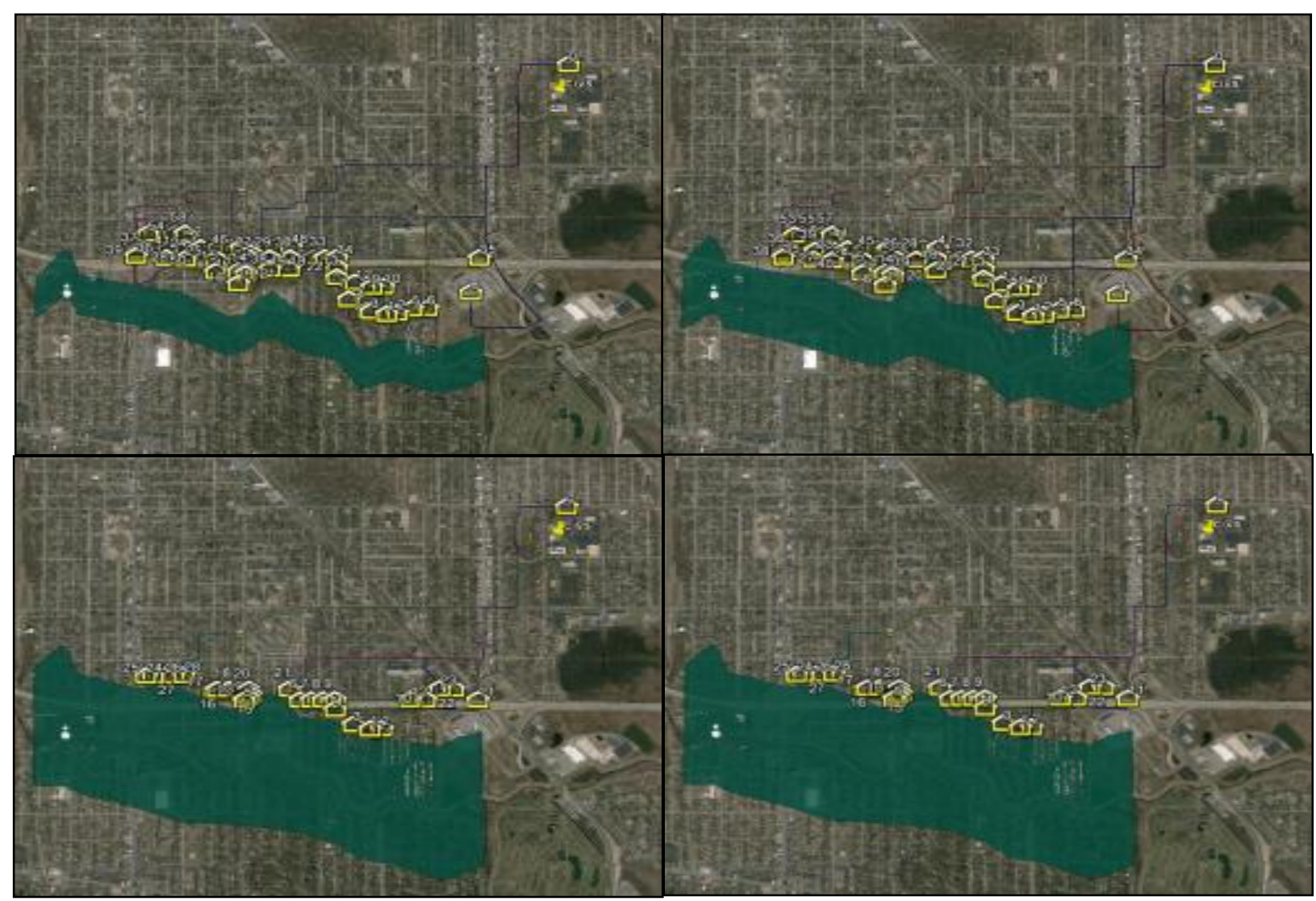

Figure 8. Real-time Update of Rescues Route with the Changes in Disaster Area

The web-based 3D visualization interface was developed in response to two specific criteria: First, that disaster resource requests and locations must be visible to users of the system; and secondly, that users of the system must have control of the type and quantity of information displayed in the user interface. The new elements of the system were designed to be backward compatible with previous system considerations. An illustration of a typical usage scenario follows: A remote user opens the visualization interface in a HTML 5 compliant web browser. The interface presented contains the main display surface based on 3D Google Earth. A number of sub-panels located around the main display surface allow the user to enable or disable elements visible within the Google Earth environment and view information about each element being displayed.

\section{Project-based Learning for Graduate and Undergraduate Education}

From year 2010, more than 30 PUC graduate and undergraduate students have participated in this research. The authors worked closely with graduate and undergraduate students in developing a web-based disaster management and communication system and distributed graph partitioning algorithm in Purdue University Calumet HPC Cluster for disaster decision support. 
During the project, the students acquired hands-on experience in grid computing as well as interactive visualization techniques. Students developed HPC based disaster decision support system to compute the effective resource distribution strategy and prioritize the rescue areas by utilizing disaster specific geospatial information system. Three graduate students completed their master's thesis based on this project, and more than 10 undergraduate students completed their senior design project based on this research.

\section{Conclusions}

This paper described the development of web based 3D Visualization and cluster computing model for the disaster management and rescue effort. The developed system is portable and selfsupporting with laptops or handheld devices that can be carried into a disaster area. The components of the system could be quickly and easily transported to any disaster terrain site. After deployment, the system could be operational and functional within just a few hours. In addition, utilization of freely available software packages proved effective as a low-cost solution to the problem. Disaster management systems are currently not installed as a consistent solution across the nation. This research will lay a foundation for disaster preparedness, management and decision support system for the local and federal government. Integration of 3D geospatial visualization and middle-sized computational cluster with disaster data management and communication system (DMCS) has the potential to provide innovative disaster management solutions to the local and federal government agencies.

This work is supported in part by the Northwest Indiana Computational Grid Project funded by Department of Energy.

\section{Bibliography}

1. U.S. Government 2006. The FEDERAL RESPONSE TO HURRICANE KATRINA: LESSONS LEARNED. http://georgewbush-whitehouse.archives.gov/reports/katrina-lessons-learned.pdf. 2008-06-06.

2. Nicolai B., Puntillo K., Bilow B. 2008. Research Design for a Natural Disaster Management Communication System: Local Indiana Government Agency Model, Proceedings of the Information Systems Education Conference 2008, v 25, Nov 6-9, Phoenix, AZ

3. Nicolai, B. 2007. Teaching Relational Database Modeling Using A Case Study: Indigent Population Database Project. Can our existing government agencies provide life support to our indigent population and survive a national disaster, Proceedings from ATINER 2007 International Conference. Athens, Greece.

4. Nicolai, Barbara, 2007. Teaching citizenship through database case study application, the Hurricane Katrina Disaster experience, International Conference on Information Society (i-Society) 2007, Oct 7-11, Merrillville, IN.

5. Nicolai, Barbara, Winer, Charles, 2006. "Indigent Population and Natural or Terrorist Database and infrastructure Project”, International Conference on Information Society (i-Society) 2006, Aug 8-14, Miami, Florida.

6. Patrick C. S.; David M. S. Technology and Communications in an Urban Crisis: The Role of Mobile Communications Systems in Disasters Journal of Urban Technology, 2009; 16(1) 133 - 149

7. Charles K.; Robert W.; William H. HAZUS Earthquake Loss Estimation Methods, Natural Hazards Rev. $20057(2), 45-59$

8. Subrahmanyam, M. Riverine Flood Modeling in HAZUS-MH: Overview of the implementation, Proceedings of the 2005 Georgia Water Resources Conference. 2005

9. Akhter, S., Chemin, Y., Aida, K., 2006. "Satellite Image Processing on Distributed Computing Environments”. HPCS Conference, Japan, 20 December 2006. 
10. S. Akhter, K. Aida, Y. Chemin, "GRASS GIS on High Performance Computing with MPI, OpenMP and Ninf-G Programming Framework", Proceeding of ISPRS 2010, Japan.

11. Roy P.S., Harish Karnatak, Sameer Saran ., 2012. "Geospatial Data Processing in Distributed Computing Environment". Computer Society of India Communication Vol. 35; No. 10, January 2012

12. Luis E. de la Torre, Irina S. Dolinskaya, Karen R. Smilowitz, "Disaster relief routing: Integrating research and practice", Socio-Economic Planning Sciences, Volume 46, Issue 1, March 2012, Pages 88-97.

13. Lecron, F., Manneback, P., Tuyttens, D.: Exploiting grid computation for solving the vehicle routing problem. In: 2010 IEEE/ACS International Conference on Computer Systems and Applications (AICCSA), pp. 1-6 (2010)

14. Cruz-Chávez, M. A., Rodríguez-León, A., Rivera-López, R., Juárez-Pérez, F., Peralta-Abarca, C., \& Martínez-Oropeza, A. (2012). Grid Platform Applied to the Vehicle Routing Problem with Time Windows for the Distribution of Products. In C. Ortiz Zezzatti, C. Chira, A. Hernandez, \& M. Basurto (Eds.) Logistics Management and Optimization through Hybrid Artificial Intelligence Systems (pp. 52-81).

15. Robert W. Floyd. Algorithm 97: Shortest path. Communication of. ACM 5, 6 (June 1962), 345. DOI $=10.1145 / 367766.368168$

16. Chris Groe, Bruce Golden, Edward Wasil. A library of local search heuristics for the vehicle routing problem . Mathematical Programming Computation, 2010; 2(2) 79-101. 